\title{
Is A Mode of Presentation of Colorectal Cancer Different According to Each Individual's Social-economic Status?
}

\author{
Chinock Cheong, Nam Kyu Kim \\ Department of Surgery, Yonsei University College of Medicine, Seoul, Korea
}

\section{See Article on Page 128-132}

Colorectal cancer (CRC) can be classified into sporadic and hereditary CRC. Sporadic cancer is the most common form of CRC, and environment factors are considered as main influencing causes. Socio-economic status can be important for its occurrence and can influence the diagnosis and the stage of presentation of CRC. However, evaluation of the socio-economic status of a patient is limited because of several established procedures, and its relationship to the stage of presentation of CRC is not clear. Previous studies were not able to establish a definitive correlation between socio-economic status and CRC $[1,2]$. In the same manner, this study showed no association between quintile and social deprivation or mortality or between quintile and presentation with M1 metastasis [3]. It only demonstrated a significant relationship between social deprivation and Dukes staging. However, Dukes staging has been replaced by a more detailed TNM staging and is no longer recommended for clinical use.

Socio-economic status differs according to race and region, which they have own economic and educational value. The mode of presentation of CRC is more affected by factors such as the patient's lifestyle rather than his or her socio-economic status itself [4]. For that reason, showing significant results in this study, which the authors compared to results according to the Indices of Deprivation with seven items, is not easy. In addition to factors related to the patient's lifestyle, the practices of the medical system, such as surveillance, may have an important impact on the initial presentation of CRC. If the national surveillance system is able to provide affordable screening for CRC, then a relationship

\section{Correspondence to: Nam Kyu Kim, M.D.}

Department of surgery, Yonsei University College of Medicine,

50-1 Yonsei-ro, Seodaemun-gu, Seoul 03722, Korea

Tel: +82-2-2228-2117, Fax: +82-2-313-8289

E-mail: namkyuk@yuhs.ac

(c) 2016 The Korean Society of Coloproctology

This is an open-access article distributed under the terms of the Creative Commons Attribution NonCommercial License (http://creativecommons.org/licenses/by-nc/4.0) which permits unrestricted noncommercial use, distribution, and reproduction in any medium, provided the original work is properly cited. between socio-economic status and CRC might not exist.

The diagnosis and treatment of CRC early through regular examination is important because the incidence of CRC has been increasing and $\mathrm{CRC}$ is now the third most common cause of cancer in men worldwide and the second in women worldwide [5]. Thus, vulnerable groups among the population and the factors that influence the incidence and the mode of presentation of CRC must be analyzed. In spite of this, until now, no studies have investigated the relationship between socio-economic status and the mode of presentation for CRC in Korea. For early diagnosis and treatment, classifying vulnerable groups according to socio-economic status may be necessary and may lead to a better clinical outcome.

\section{CONFLICT OF INTEREST}

No potential conflict of interest relevant to this article was reported.

\section{REFERENCES}

1. Siegel RL, Jemal A, Thun MJ, Hao Y, Ward EM. Trends in the incidence of colorectal cancer in relation to county-level poverty among blacks and whites. J Natl Med Assoc 2008;100:1441-4.

2. Kim D, Masyn KE, Kawachi I, Laden F, Colditz GA. Neighborhood socioeconomic status and behavioral pathways to risks of colon and rectal cancer in women. Cancer 2010;116:4187-96.

3. ELHadi A, Ashford-Wilson S, Brown S, Pal A, Lal R, Aryal K. Effect of social deprivation on the stage and mode of presentation of colorectal cancer. Ann Coloproctol 2016;32:128-32.

4. Kim DH. Risk factors of colorectal cancer. J Korean Soc Coloproctol 2009;25:356-62.

5. Ferlay J, Soerjomataram I, Dikshit R, Eser S, Mathers C, Rebelo M, et al. Cancer incidence and mortality worldwide: sources, methods and major patterns in GLOBOCAN 2012. Int J Cancer 2015; 136:E359-86. 